\title{
Some reverse mean inequalities for operators and matrices
}

\author{
Chaojun Yang ${ }^{1}$, Yaxin Gao ${ }^{1}$ and Fangyan Lu ${ }^{1 *}$
}

\author{
"Correspondence: fylu@suda.edu.cn \\ 1 Department of Mathematics, \\ Soochow University, Suzhou, \\ P.R. China
}

\begin{abstract}
In this paper, we present some new reverse arithmetic-geometric mean inequalities for operators and matrices due to Lin (Stud. Math. 215:187-194, 2013). Among other inequalities, we prove that if $A, B \in B(\mathcal{H})$ are accretive and $0<m l \leq \Re(A), \Re(B) \leq M l$, then, for every positive unital linear map $\Phi$,

$$
\Phi^{2}\left(\Re\left(\frac{A+B}{2}\right)\right) \leq(K(h))^{2} \Phi^{2}(\Re(A \sharp B))
$$

where $K(h)=\frac{(h+1)^{2}}{4 h}$ and $h=\frac{M}{m}$. Moreover, some reverse harmonic-geometric mean inequalities are also presented.
\end{abstract}

MSC: Primary 47A63; secondary 15A15

Keywords: Positive linear maps; Arithmetic-geometric-harmonic mean; Sector matrix; Inequality

\section{Introduction}

Throughout this paper, $B(\mathcal{H})$ stands for all bounded linear operators on a complex Hilbert space $\mathcal{H}$. In the finite-dimensional setting, $\mathbb{M}_{n}$ denotes the set of all $n \times n$ complex matrices. For $A, B \in B(\mathcal{H})$, we use $A \geq B(B \leq A)$ to mean that $A-B$ is positive. The operator norm is denoted by $\|\cdot\|$. An operator $A \in B(\mathcal{H})$ is called accretive if in its Cartesian (or Toeptliz) decomposition, $A=\Re A+i \Im A, \Re A$ is positive, where $\Re A=\frac{A+A^{*}}{2}, \Im A=\frac{A-A^{*}}{2 i}$. A linear map $\Phi: B(\mathcal{H}) \rightarrow B(\mathcal{H})$ is called positive if $\Phi(A) \geq 0$ whenever $A \geq 0$. If $\Phi(I)=I$, where $I$ denotes the identity operator, then we say that $\Phi$ is unital. We reserve $M, m$ for scalars. In the finite-dimensional setting, we use $I_{n}$ for the identity.

The numerical range of $A \in \mathbb{M}_{n}$ is defined by

$$
W(A)=\left\{x^{*} A x: x \in \mathbb{C}^{n}, x^{*} x=1\right\}
$$

For $\alpha \in\left[0, \frac{\pi}{2}\right), S_{\alpha}$ denote the sector regions in the complex plane as follows:

$$
S_{\alpha}=\{z \in \mathbb{C}: \Re z \geq 0,|\Im z| \leq(\Re z) \tan \alpha\} .
$$

Clearly, $A$ is positive semidefinite if and only if $W(A) \subset S_{0}$, and if $W(A), W(B) \subset S_{\alpha}$ for some $\alpha \in\left[0, \frac{\pi}{2}\right)$, then $W(A+B) \subset S_{\alpha}$. As $0 \notin S_{\alpha}$, if $W(A) \subset S_{\alpha}$, then $A$ is nonsingu-

(c) The Author(s) 2019. This article is distributed under the terms of the Creative Commons Attribution 4.0 International License (http://creativecommons.org/licenses/by/4.0/), which permits unrestricted use, distribution, and reproduction in any medium, provided you give appropriate credit to the original author(s) and the source, provide a link to the Creative Commons license, and indicate if changes were made. 
lar. Moreover, $W(A) \subset S_{\alpha}$ implies $W\left(X^{*} A X\right) \subset S_{\alpha}$ for any nonzero $n \times m$ matrix $X$, thus $W\left(A^{-1}\right) \subset S_{\alpha}$.

Recent studies on matrices with numerical ranges in a sector can be found in $[3-5,8-10$, $14]$ and the references therein.

In [13], Tominaga presented an operator inequality as follows: Let $A, B$ be positive operators on a Hilbert space with $0<m I \leq A, B \leq M I$, then

$$
\frac{A+B}{2} \leq S(h) A \sharp B,
$$

where $S(h)=\frac{\frac{1}{h-1}}{e \log h \frac{1}{h-1}}$ is called Specht's ratio and $h=\frac{M}{m}$.

Lin [6] found that, for a positive unital linear map $\Phi$ between $C^{*}$-algebra,

$$
\Phi\left(\frac{A+B}{2}\right) \leq K(h) \Phi(A \sharp B)
$$

due to (1) and the following observation [6]:

$$
S(h) \leq K(h) \leq S^{2}(h) \quad(h \geq 1),
$$

where $K(h)=\frac{(h+1)^{2}}{4 h}$.

It is well known that, for two general positive operators (or positive definite matrices) $A, B$,

$$
A \geq B \quad \nRightarrow \quad A^{2} \geq B^{2} .
$$

However, Lin [6] showed that (2) can be squared as follows:

$$
\Phi^{2}\left(\frac{A+B}{2}\right) \leq K^{2}(h) \Phi^{2}(A \sharp B) .
$$

Zhang [15] generalized (3) when $p \geq 2$ as follows:

$$
\Phi^{2 p}\left(\frac{A+B}{2}\right) \leq \frac{\left(K(h)\left(M^{2}+m^{2}\right)\right)^{2 p}}{16 M^{2 p} m^{2 p}} \Phi^{2 p}(A \sharp B) .
$$

For two accretive operators $A, B \in B(\mathcal{H})$, Drury [3] defined the geometric mean of $A$ and $B$ as follows:

$$
A \sharp B=\left(\frac{2}{\pi} \int_{0}^{\infty}\left(t A+t^{-1} B\right)^{-1} \frac{d t}{t}\right)^{-1} .
$$

This new geometric mean defined by (5) possesses some similar properties compared to the geometric mean of two positive operators. For instance, $A \sharp B=B \sharp A,(A \sharp B)^{-1}=$ $A^{-1} \sharp B^{-1}$. For more information about the geometric mean of two accretive operators, see [3]. Moreover, if $A, B \in \mathbb{M}_{n}$ with $W(A), W(B) \subset S_{\alpha}$, then $W(A \sharp B) \subset S_{\alpha}$.

Following an idea of Lin [6], we shall give some new reverse arithmetic-geometric mean inequalities for operators and matrices which can be seen as a complementary of (3) and (4). Moreover, some reverse harmonic-geometric mean inequalities are also presented. 


\section{Main results}

To reach our goal, we need the following lemmas.

Lemma 2.1 ([10]) If $A, B \in B(\mathcal{H})$ are accretive, then

$$
\Re(A) \sharp \Re(B) \leq \Re(A \sharp B) .
$$

Lemma 2.2 ([10]) If $A, B \in B(\mathcal{H})$ are accretive, then

$$
\Re\left(\left(\frac{A^{-1}+B^{-1}}{2}\right)^{-1}\right) \geq\left(\frac{\Re(A)^{-1}+\Re(B)^{-1}}{2}\right)^{-1} .
$$

Lemma 2.3 ([9]) If $A \in \mathbb{M}_{n}$ has a positive definite real part, then

$$
\Re\left(A^{-1}\right) \leq \Re(A)^{-1} .
$$

Lemma 2.4 ([4]) If $A \in \mathbb{M}_{n}$ with $W(A) \subset S_{\alpha}$, then

$$
\sec ^{2}(\alpha) \Re\left(A^{-1}\right) \geq \Re(A)^{-1} .
$$

It is easy to verify that $\Re\left(\left(\frac{A^{-1}+B^{-1}}{2}\right)^{-1}\right) \leq \Re(A \sharp B) \leq \Re\left(\frac{A+B}{2}\right)$ does not persist for two accretive operators $A$ and $B$. However, Lin presented the following extension of the arithmeticgeometric mean inequality.

Lemma 2.5 ([9]) Let $A, B \in \mathbb{M}_{n}$ be such that $W(A)$, $W(B) \subset S_{\alpha}$. Then

$$
\Re(A \sharp B) \leq \sec ^{2}(\alpha) \Re\left(\frac{A+B}{2}\right) .
$$

Lemma 2.6 ([2]) Let $A, B \in B(\mathcal{H})$ be positive. Then

$$
\|A B\| \leq \frac{1}{4}\|A+B\|^{2} .
$$

Lemma 2.7 ([1]) Let $A \in B(\mathcal{H})$ be positive. Then, for every positive unital linear map $\Phi$,

$$
\Phi^{-1}(A) \leq \Phi\left(A^{-1}\right)
$$

Lemma 2.8 ([1]) Let $A, B \in B(\mathcal{H})$ be positive. Then, for $1 \leq r<+\infty$,

$$
\left\|A^{r}+B^{r}\right\| \leq\left\|(A+B)^{r}\right\|
$$

An operator Kantorovich inequality obtained by Marshall and Olkin [12] reads as follows.

Let $0<m I \leq A \leq M I$, then, for every positive unital linear map $\Phi$,

$$
\Phi\left(A^{-1}\right) \leq K(h) \Phi(A)^{-1},
$$

where $K(h)=\frac{(h+1)^{2}}{4 h}$ and $h=\frac{M}{m}$. 
Lin [7] showed that (7) can be squared as follows:

$$
\Phi^{2}\left(A^{-1}\right) \leq(K(h))^{2} \Phi(A)^{-2}
$$

where $K(h)=\frac{(h+1)^{2}}{4 h}$ and $h=\frac{M}{m}$.

Let $A \in \mathbb{M}_{n}$ have a positive definite real part, $0<m I_{n} \leq \Re(A) \leq M I_{n}$ and $\Phi$ be a unital positive linear map. By (7) and Lemma 2.3, we can obtain the following inequality:

$$
\Phi\left(\Re\left(A^{-1}\right)\right) \leq K(h) \Phi(\Re(A))^{-1},
$$

where $K(h)=\frac{(h+1)^{2}}{4 h}$ and $h=\frac{M}{m}$.

As an analog of inequality (8), we show that inequality (9) can be squared nicely as follows.

Theorem 2.9 If $A \in \mathbb{M}_{n}$ has a positive definite real part and $0<m I_{n} \leq \Re(A) \leq M I_{n}$, then, for every positive unital linear map $\Phi$,

$$
\Phi^{2}\left(\Re\left(A^{-1}\right)\right) \leq(K(h))^{2} \Phi(\Re(A))^{-2},
$$

where $K(h)=\frac{(h+1)^{2}}{4 h}$ and $h=\frac{M}{m}$.

Proof Since

$$
m I_{n} \leq \Re(A) \leq M I_{n},
$$

we have

$$
\left(M I_{n}-\Re(A)\right)\left(m I_{n}-\Re(A)\right) \Re(A)^{-1} \leq 0,
$$

which is equivalent to

$$
\Re(A)+M m \Re(A)^{-1} \leq(M+m) I_{n} .
$$

By Lemma 2.3 and (11), we get

$$
\begin{aligned}
\Re(A) & +M m \Re\left(A^{-1}\right) \\
\leq & \Re(A)+M m \Re(A)^{-1} \\
\leq & (M+m) I_{n} .
\end{aligned}
$$

Inequality (10) is equivalent to

$$
\left\|\Phi\left(\Re\left(A^{-1}\right)\right) \Phi(\Re(A))\right\| \leq K(h) .
$$

By computation, we have

$$
\left\|M m \Phi\left(\Re\left(A^{-1}\right)\right) \Phi(\Re(A))\right\|
$$




$$
\begin{aligned}
& \leq \frac{1}{4}\left\|M m \Phi\left(\Re\left(A^{-1}\right)\right)+\Phi(\Re(A))\right\|^{2} \quad \text { (by Lemma 2.6) } \\
& \leq \frac{1}{4}(M+m)^{2} \quad(\text { by }(12))
\end{aligned}
$$

That is,

$$
\left\|\Phi\left(\Re\left(A^{-1}\right)\right) \Phi(\Re(A))\right\| \leq K(h) .
$$

This completes the proof.

Let $A, B \in B(\mathcal{H})$ be accretive, $0<m I \leq \Re(A), \Re(B) \leq M I$ and $\Phi$ be a unital positive linear map. By inequality (2) and Lemma 2.1, we can obtain the following inequality:

$$
\Phi\left(\Re\left(\frac{A+B}{2}\right)\right) \leq K(h) \Phi(\Re(A \sharp B)),
$$

where $K(h)=\frac{(h+1)^{2}}{4 h}$ and $h=\frac{M}{m}$.

Following an idea of Lin [6], we give a squaring version of inequality (13) below.

Theorem 2.10 If $A, B \in \mathbb{M}_{n}$ with $W(A), W(B) \subset S_{\alpha}$ and $0<m I_{n} \leq \Re(A), \Re(B) \leq M I_{n}$, then, for every positive unital linear map $\Phi$,

$$
\Phi^{2}\left(\Re\left(\frac{A+B}{2}\right)\right) \leq\left(\sec ^{4}(\alpha) K(h)\right)^{2} \Phi^{2}(\Re(A \sharp B)),
$$

where $K(h)=\frac{(h+1)^{2}}{4 h}$ and $h=\frac{M}{m}$.

Proof From Theorem 2.9 we have

$$
\frac{1}{2} \Re(A)+\frac{1}{2} M m \Re(A)^{-1} \leq \frac{1}{2}(M+m) I_{n}
$$

and

$$
\frac{1}{2} \Re(B)+\frac{1}{2} M m \Re(B)^{-1} \leq \frac{1}{2}(M+m) I_{n} .
$$

Summing up inequalities (15) and (16), we get

$$
\Re\left(\frac{A+B}{2}\right)+M m\left(\frac{\Re(A)^{-1}+\Re(B)^{-1}}{2}\right) \leq(M+m) I_{n} .
$$

Inequality (14) is equivalent to

$$
\left\|\Phi\left(\Re\left(\frac{A+B}{2}\right)\right) \Phi^{-1}(\Re(A \sharp B))\right\| \leq \sec ^{4}(\alpha) K(h) .
$$

By computation, we have

$$
\left\|\sec ^{4}(\alpha) M m \Phi\left(\Re\left(\frac{A+B}{2}\right)\right) \Phi^{-1}(\Re(A \sharp B))\right\|
$$




$$
\begin{aligned}
& \leq \frac{1}{4}\left\|\sec ^{4}(\alpha) \Phi\left(\Re\left(\frac{A+B}{2}\right)\right)+M m \Phi^{-1}(\Re(A \sharp B))\right\|^{2} \quad \text { (by Lemma 2.6) } \\
& \leq \frac{1}{4}\left\|\sec ^{4}(\alpha) \Phi\left(\Re\left(\frac{A+B}{2}\right)\right)+M m \Phi\left((\Re(A \sharp B))^{-1}\right)\right\|^{2} \quad \text { (by Lemma 2.7) } \\
& \leq \frac{1}{4}\left\|\sec ^{4}(\alpha) \Phi\left(\Re\left(\frac{A+B}{2}\right)\right)+\sec ^{2}(\alpha) M m \Phi\left(\Re\left(A^{-1} \sharp B^{-1}\right)\right)\right\|^{2} \quad \text { (by Lemma 2.4) } \\
& \leq \frac{1}{4}\left\|\sec ^{4}(\alpha) \Phi\left(\Re\left(\frac{A+B}{2}\right)\right)+\sec ^{4}(\alpha) M m \Phi\left(\mathfrak{R}\left(\frac{A^{-1}+B^{-1}}{2}\right)\right)\right\|^{2} \quad \text { (by (6)) } \\
& =\frac{1}{4}\left\|\sec ^{4}(\alpha) \Phi\left(\mathfrak{R}\left(\frac{A+B}{2}\right)+M m \Re\left(\frac{A^{-1}+B^{-1}}{2}\right)\right)\right\|^{2} \quad \text { (by Lemma 2.3) } \\
& \leq \frac{1}{4} \| \sec ^{4}(\alpha) \Phi\left(\Re\left(\frac{A+B}{2}\right)+M m\left(\frac{\Re(A)^{-1}+\Re(B)^{-1}}{2}\right) \|^{2} \quad\right. \\
& \leq \frac{1}{4} \sec ^{8}(\alpha)(M+m)^{2} \quad(\text { by }(17)) .
\end{aligned}
$$

That is,

$$
\left\|\Phi\left(\Re\left(\frac{A+B}{2}\right)\right) \Phi^{-1}(\Re(A \sharp B))\right\| \leq \sec ^{4}(\alpha) K(h) .
$$

This completes the proof.

Next we give a $p$ th $(p \geq 2)$ powering of inequality (14).

Theorem 2.11 If $A, B \in \mathbb{M}_{n}$ with $W(A), W(B) \subset S_{\alpha}, 0<m I_{n} \leq \Re(A), \Re(B) \leq M I_{n}, 1<\beta \leq$ 2 and $p \geq 2 \beta$, then, for every positive unital linear map $\Phi$,

$$
\Phi^{p}\left(\Re\left(\frac{A+B}{2}\right)\right) \leq \frac{\left(\sec ^{2 \beta}(\alpha) K(h)^{\frac{\beta}{2}}\left(M^{\beta}+m^{\beta}\right)\right)^{\frac{2 p}{\beta}}}{16 M^{p} m^{p}} \Phi^{p}(\Re(A \sharp B)),
$$

where $K(h)=\frac{(h+1)^{2}}{4 h}$ and $h=\frac{M}{m}$.

Proof Since

$$
m I_{n} \leq \Phi\left(\Re\left(\frac{A+B}{2}\right)\right) \leq M I_{n}
$$

we have

$$
M^{\beta} m^{\beta} \Phi^{-\beta}\left(\Re\left(\frac{A+B}{2}\right)\right)+\Phi^{\beta}\left(\Re\left(\frac{A+B}{2}\right)\right) \leq M^{\beta}+m^{\beta} .
$$

By (14) and the L-H inequality [1], we obtain

$$
\Phi^{-\beta}(\Re(A \sharp B)) \leq\left(\sec ^{4}(\alpha) K(h)\right)^{\beta} \Phi^{-\beta}\left(\Re\left(\frac{A+B}{2}\right)\right) .
$$


Inequality (18) is equivalent to

$$
\left\|\Phi^{\frac{p}{2}}\left(\Re\left(\frac{A+B}{2}\right)\right) \Phi^{-\frac{p}{2}}(\Re(A \sharp B))\right\| \leq \frac{\left(\sec ^{2 \beta}(\alpha) K(h)^{\frac{\beta}{2}}\left(M^{\beta}+m^{\beta}\right)\right)^{\frac{p}{\beta}}}{4 M^{\frac{p}{2}} m^{\frac{p}{2}}} .
$$

By computation, we have

$$
\begin{aligned}
\| M^{\frac{p}{2}} & m^{\frac{p}{2}} \Phi^{\frac{p}{2}}\left(\Re\left(\frac{A+B}{2}\right)\right) \Phi^{-\frac{p}{2}}(\Re(A \sharp B)) \| \\
\leq & \frac{1}{4}\left\|\left(\sec ^{4}(\alpha) K(h)\right)^{\frac{p}{4}} \Phi^{\frac{p}{2}}\left(\Re\left(\frac{A+B}{2}\right)\right)+\left(\frac{M^{2} m^{2}}{\sec ^{4}(\alpha) K(h)}\right)^{\frac{p}{4}} \Phi^{-\frac{p}{2}}(\Re(A \sharp B))\right\|^{2} \\
\leq & \frac{1}{4}\left\|\left(\sec ^{4}(\alpha) K(h)\right)^{\frac{\beta}{2}} \Phi^{\beta}\left(\Re\left(\frac{A+B}{2}\right)\right)+\left(\frac{M^{2} m^{2}}{\sec ^{4}(\alpha) K(h)}\right)^{\frac{\beta}{2}} \Phi^{-\beta}(\Re(A \sharp B))\right\|^{\frac{p}{\beta}} \\
\leq & \frac{1}{4} \|\left(\sec ^{4}(\alpha) K(h)\right)^{\frac{\beta}{2}} \Phi^{\beta}\left(\Re\left(\frac{A+B}{2}\right)\right) \\
& +\left(\sec ^{4}(\alpha) K(h)\right)^{\frac{\beta}{2}} M^{\beta} m^{\beta} \Phi^{-\beta}\left(\Re\left(\frac{A+B}{2}\right)\right) \|^{\frac{p}{\beta}} \\
= & \frac{1}{4}\left\|\left(\sec ^{4}(\alpha) K(h)\right)^{\frac{\beta}{2}}\left(\Phi^{\beta}\left(\Re\left(\frac{A+B}{2}\right)\right)+M^{\beta} m^{\beta} \Phi^{-\beta}\left(\Re\left(\frac{A+B}{2}\right)\right)\right)\right\|^{\frac{p}{\beta}} \\
\leq & \frac{1}{4}\left(\sec ^{2 \beta}(\alpha) K(h)^{\frac{\beta}{2}}\left(M^{\beta}+m^{\beta}\right)\right)^{\frac{p}{\beta}},
\end{aligned}
$$

where the first inequality is by Lemma 2.6 , the second one is by Lemma 2.8 , the third one is by (20) and the last one is by (19).

That is,

$$
\left\|\Phi^{\frac{p}{2}}\left(\Re\left(\frac{A+B}{2}\right)\right) \Phi^{-\frac{p}{2}}(\Re(A \sharp B))\right\| \leq \frac{\left(\sec ^{2 \beta}(\alpha) K(h)^{\frac{\beta}{2}}\left(M^{\beta}+m^{\beta}\right)\right)^{\frac{p}{\beta}}}{4 M^{\frac{p}{2}} m^{\frac{p}{2}}} .
$$

This completes the proof.

We are not satisfied with the factor $\left(\sec ^{4}(\alpha) K(h)\right)^{2}$ in Theorem 2.10, the ideal factor should be $(K(h))^{2}$. We shall prove it in the following theorem.

Theorem 2.12 If $A, B \in B(\mathcal{H})$ are accretive and $0<m I \leq \Re(A), \Re(B) \leq M I$, then, for every positive unital linear map $\Phi$,

$$
\Phi^{2}\left(\Re\left(\frac{A+B}{2}\right)\right) \leq(K(h))^{2} \Phi^{2}(\Re(A \sharp B)),
$$

where $K(h)=\frac{(h+1)^{2}}{4 h}$ and $h=\frac{M}{m}$.

Proof From Theorem 2.10 one can get

$$
\Re\left(\frac{A+B}{2}\right)+M m\left(\frac{\Re(A)^{-1}+\Re(B)^{-1}}{2}\right) \leq(M+m) I .
$$


Inequality (21) is equivalent to

$$
\left\|\Phi\left(\Re\left(\frac{A+B}{2}\right)\right) \Phi^{-1}(\Re(A \sharp B))\right\| \leq K(h) .
$$

By computation, we have

$$
\begin{aligned}
& \left\|\operatorname{Mm} \Phi\left(\Re\left(\frac{A+B}{2}\right)\right) \Phi^{-1}(\Re(A \not B))\right\| \\
& \leq \frac{1}{4}\left\|\Phi\left(\Re\left(\frac{A+B}{2}\right)\right)+M m \Phi^{-1}(\Re(A \sharp B))\right\|^{2} \quad \text { (by Lemma 2.6) } \\
& \leq \frac{1}{4}\left\|\Phi\left(\Re\left(\frac{A+B}{2}\right)\right)+M m \Phi\left((\Re(A \sharp B))^{-1}\right)\right\|^{2} \quad \text { (by Lemma 2.7) } \\
& \leq \frac{1}{4}\left\|\Phi\left(\Re\left(\frac{A+B}{2}\right)\right)+M m \Phi\left((\Re(A) \sharp \Re(B))^{-1}\right)\right\|^{2} \quad \text { (by Lemma 2.1) } \\
& =\frac{1}{4}\left\|\Phi\left(\Re\left(\frac{A+B}{2}\right)\right)+\operatorname{Mm} \Phi\left(\Re(A)^{-1} \sharp \Re(B)^{-1}\right)\right\|^{2} \\
& \leq \frac{1}{4}\left\|\Phi\left(\Re\left(\frac{A+B}{2}\right)\right)+\operatorname{Mm} \Phi\left(\frac{\Re(A)^{-1}+\Re(B)^{-1}}{2}\right)\right\|^{2} \quad \text { (by AM-GM inequality) } \\
& \left.\leq \frac{1}{4}(M+m)^{2} \quad \text { (by }(22)\right) \text {. }
\end{aligned}
$$

That is,

$$
\left\|\Phi\left(\Re\left(\frac{A+B}{2}\right)\right) \Phi^{-1}(\Re(A \sharp B))\right\| \leq K(h) .
$$

This completes the proof.

Remark 2.13 Letting $A, B \geq 0$ in Theorem 2.12, inequality (21) coincides with inequality (3).

Next we give a $p$ th $(p \geq 2)$ powering of inequality (21) along the same line as in Theorem 2.11.

Theorem 2.14 If $A, B \in B(\mathcal{H})$ are accretive and $0<m I \leq \Re(A), \Re(B) \leq M I 1<\beta \leq 2$ and $p \geq 2 \beta$, then, for every positive unital linear map $\Phi$,

$$
\Phi^{p}\left(\Re\left(\frac{A+B}{2}\right)\right) \leq \frac{\left(K(h)^{\frac{\beta}{2}}\left(M^{\beta}+m^{\beta}\right)\right)^{\frac{2 p}{\beta}}}{16 M^{p} m^{p}} \Phi^{p}(\Re(A \sharp B)),
$$

where $K(h)=\frac{(h+1)^{2}}{4 h}$ and $h=\frac{M}{m}$.

Remark 2.15 Letting $A, B \geq 0$ and $\beta=2$ in Theorem 2.14, inequality (23) coincides with inequality (4).

The following theorem corrects Theorem 1.2 of Liu et al. [11]. 
Theorem 2.16 Let $A, B \in \mathbb{M}_{n}$ be such that $W(A), W(B) \subset S_{\alpha}$, then

$$
\Re\left(\left(\frac{A^{-1}+B^{-1}}{2}\right)^{-1}\right) \leq \sec ^{4}(\alpha) \Re(A \sharp B) .
$$

Proof We can get

$$
\left(\Re\left(\frac{A^{-1}+B^{-1}}{2}\right)\right)^{-1} \leq \sec ^{2}(\alpha)\left(\Re\left(A^{-1}\right) \sharp \Re\left(B^{-1}\right)\right)^{-1}
$$

along the same line as Liu et al. did in [11] by Lemma 2.1 and Lemma 2.5 .

Thus we have

$$
\begin{aligned}
\Re\left(\left(\frac{A^{-1}+B^{-1}}{2}\right)^{-1}\right) & \leq\left(\Re\left(\frac{A^{-1}+B^{-1}}{2}\right)\right)^{-1} \quad \text { (by Lemma 2.3) } \\
& \leq \sec ^{2}(\alpha)\left(\Re\left(A^{-1}\right) \sharp \Re\left(B^{-1}\right)\right)^{-1} \quad(\text { by }(25)) \\
& =\sec ^{2}(\alpha)\left(\Re\left(A^{-1}\right)\right)^{-1} \sharp\left(\Re\left(B^{-1}\right)\right)^{-1} \\
& \leq \sec ^{4}(\alpha)(\Re(A) \sharp \Re(B)) \quad(\text { by Lemma 2.4) } \\
& \leq \sec ^{4}(\alpha) \Re(A \sharp B) \quad \text { (by Lemma 2.1). }
\end{aligned}
$$

This completes the proof.

Remark 2.17 Maybe it is just a clerical error in Theorem 1.2 of their work [11]. However, the authors present the following inequalities in their proof:

$$
\begin{aligned}
\Re\left(\left(\frac{A^{-1}+B^{-1}}{2}\right)^{-1}\right) & \leq \sec ^{2}(\alpha)\left(\Re\left(A^{-1}\right)\right)^{-1} \sharp\left(\Re\left(B^{-1}\right)\right)^{-1} \\
& \leq \sec ^{2}(\alpha)(\Re(A) \sharp \Re(B)) .
\end{aligned}
$$

Obviously, such a deduction in their proof collapses given the property of geometric mean for positive definite matrices. Thus we give Theorem 2.16 and the proof.

Let $A, B \in \mathbb{M}_{n}$ with $W(A), W(B) \subset S_{\alpha}, 0<m I_{n} \leq \Re\left(A^{-1}\right), \Re\left(B^{-1}\right) \leq M I_{n}$ and $\Phi$ be a unital positive linear map. As a complement of inequalities (13) and (24), we have the following reverse harmonic-geometric mean inequality:

$$
\Phi(\Re(A \sharp B)) \leq \sec ^{2}(\alpha) K(h) \Phi\left(\Re\left(\left(\frac{A^{-1}+B^{-1}}{2}\right)^{-1}\right)\right),
$$

where $K(h)=\frac{(h+1)^{2}}{4 h}$ and $h=\frac{M}{m}$.

Proof Compute

$$
\begin{aligned}
\Re(A \sharp B) & =\Re\left(\left(A^{-1} \sharp B^{-1}\right)^{-1}\right) \\
& \leq \Re\left(A^{-1} \sharp B^{-1}\right)^{-1} \\
& \leq K(h) \Re\left(\frac{A^{-1}+B^{-1}}{2}\right)^{-1}
\end{aligned}
$$




$$
\leq \sec ^{2}(\alpha) K(h) \Re\left(\left(\frac{A^{-1}+B^{-1}}{2}\right)^{-1}\right),
$$

in which the first inequality is by Lemma 2.3, the second one is by inequality (13) and the last one is by Lemma 2.4 .

Imposing $\Phi$ on both sides of the inequalities above, we thus obtain inequality (26).

As an analog of Theorem 2.12, we shall present a squaring version of inequality (26).

Theorem 2.18 If $A, B \in \mathbb{M}_{n}$ with $W(A), W(B) \subset S_{\alpha}$ and $0<m I_{n} \leq \Re\left(A^{-1}\right), \Re\left(B^{-1}\right) \leq M I_{n}$, then, for every positive unital linear map $\Phi$,

$$
\Phi^{2}(\Re(A \sharp B)) \leq\left(\sec ^{4}(\alpha) K(h)\right)^{2} \Phi^{2}\left(\Re\left(\left(\frac{A^{-1}+B^{-1}}{2}\right)^{-1}\right)\right),
$$

where $K(h)=\frac{(h+1)^{2}}{4 h}$ and $h=\frac{M}{m}$.

Proof From Theorem 2.10 we have

$$
\frac{1}{2} \Re\left(A^{-1}\right)+\frac{1}{2} M m \Re\left(A^{-1}\right)^{-1} \leq \frac{1}{2}(M+m) I_{n}
$$

and

$$
\frac{1}{2} \Re\left(B^{-1}\right)+\frac{1}{2} M m \Re\left(B^{-1}\right)^{-1} \leq \frac{1}{2}(M+m) I_{n} .
$$

Summing up inequalities (28) and (29), we get

$$
\begin{aligned}
& \Re\left(\frac{A^{-1}+B^{-1}}{2}\right)+M m \Re\left(\frac{A+B}{2}\right) \\
& \quad \leq \Re\left(\frac{A^{-1}+B^{-1}}{2}\right)+M m\left(\frac{\Re\left(A^{-1}\right)^{-1}+\Re\left(B^{-1}\right)^{-1}}{2}\right) \\
& \quad \leq(M+m) I_{n} .
\end{aligned}
$$

Inequality (27) is equivalent to

$$
\left\|\Phi(\Re(A \sharp B)) \Phi^{-1}\left(\Re\left(\left(\frac{A^{-1}+B^{-1}}{2}\right)^{-1}\right)\right)\right\| \leq \sec ^{4}(\alpha) K(h) .
$$

By computation, we have

$$
\begin{aligned}
\| & M m \Phi(\Re(A \sharp B)) \Phi^{-1}\left(\Re\left(\left(\frac{A^{-1}+B^{-1}}{2}\right)^{-1}\right)\right) \| \\
\leq & \frac{1}{4}\left\|M m \Phi(\Re(A \sharp B))+\Phi^{-1}\left(\Re\left(\left(\frac{A^{-1}+B^{-1}}{2}\right)^{-1}\right)\right)\right\|^{2} \quad \text { (by Lemma 2.6) } \\
\leq & \frac{1}{4}\left\|M m \Phi(\Re(A \sharp B))+\Phi\left(\Re\left(\left(\frac{A^{-1}+B^{-1}}{2}\right)^{-1}\right)^{-1}\right)\right\|^{2} \quad \text { (by Lemma 2.7) } \\
\leq & \frac{1}{4}\left\|M m \Phi(\Re(A \sharp B))+\sec ^{2}(\alpha) \Phi\left(\Re\left(\frac{A^{-1}+B^{-1}}{2}\right)\right)\right\|^{2} \quad \text { (by Lemma 2.4) }
\end{aligned}
$$




$$
\begin{aligned}
& \leq \frac{1}{4}\left\|\sec ^{2}(\alpha) \operatorname{Mm} \Phi\left(\Re\left(\frac{A+B}{2}\right)\right)+\sec ^{2}(\alpha) \Phi\left(\Re\left(\frac{A^{-1}+B^{-1}}{2}\right)\right)\right\|^{2} \quad \text { (by (6)) } \\
& =\frac{1}{4}\left\|\sec ^{2}(\alpha) \Phi\left(M m \Re\left(\frac{A+B}{2}\right)+\Re\left(\frac{A^{-1}+B^{-1}}{2}\right)\right)\right\|^{2} \\
& \leq \frac{1}{4} \sec ^{4}(\alpha)(M+m)^{2} .
\end{aligned}
$$

That is,

$$
\left\|\Phi(\Re(A \sharp B)) \Phi^{-1}\left(\Re\left(\left(\frac{A^{-1}+B^{-1}}{2}\right)^{-1}\right)\right)\right\| \leq \sec ^{4}(\alpha) K(h) .
$$

This completes the proof.

Obviously, the optimal factor in Theorem 2.18 should be $\left(\sec ^{2}(\alpha) K(h)\right)^{2}$. We note that it is affirmative under the condition $m I_{n} \leq \Re\left(A^{-1}\right) \leq \Re(A)^{-1} \leq M I_{n}$ and $m I_{n} \leq \Re\left(B^{-1}\right) \leq$ $\Re(B)^{-1} \leq M I_{n}$ by presenting the following theorem.

Theorem 2.19 If $A, B \in \mathbb{M}_{n}$ with $W(A), W(B) \subset S_{\alpha}$ and $0<m I_{n} \leq \Re(A)^{-1}, \Re(B)^{-1} \leq M I_{n}$, then, for every positive unital linear map $\Phi$,

$$
\Phi^{2}(\Re(A \sharp B)) \leq\left(\sec ^{2}(\alpha) K(h)\right)^{2} \Phi^{2}\left(\Re\left(\left(\frac{A^{-1}+B^{-1}}{2}\right)^{-1}\right)\right),
$$

where $K(h)=\frac{(h+1)^{2}}{4 h}$ and $h=\frac{M}{m}$.

Proof From Theorem 2.10 we obtain

$$
\frac{\Re(A)^{-1}+\Re(B)^{-1}}{2}+M m \Re\left(\frac{A+B}{2}\right) \leq(M+m) I_{n} .
$$

Inequality (30) is equivalent to

$$
\left\|\Phi(\Re(A \sharp B)) \Phi^{-1}\left(\Re\left(\left(\frac{A^{-1}+B^{-1}}{2}\right)^{-1}\right)\right)\right\| \leq \sec ^{2}(\alpha) K(h) .
$$

By computation, we have

$$
\begin{aligned}
\| & \sec ^{2}(\alpha) M m \Phi(\Re(A \sharp B)) \Phi^{-1}\left(\Re\left(\left(\frac{A^{-1}+B^{-1}}{2}\right)^{-1}\right)\right) \| \\
& \leq \frac{1}{4}\left\|M m \Phi(\Re(A \sharp B))+\sec ^{2}(\alpha) \Phi^{-1}\left(\Re\left(\left(\frac{A^{-1}+B^{-1}}{2}\right){ }^{-1}\right)\right)\right\|^{2} \quad \text { (by Lemma 2.6) } \\
& \leq \frac{1}{4}\left\|M m \Phi(\Re(A \sharp B))+\sec ^{2}(\alpha) \Phi\left(\Re\left(\left(\frac{A^{-1}+B^{-1}}{2}\right)^{-1}\right)^{-1}\right)\right\|^{2} \quad \text { (by Lemma 2.7) } \\
& \leq \frac{1}{4}\left\|\operatorname{Mm} \Phi(\Re(A \sharp B))+\sec ^{2}(\alpha) \Phi\left(\frac{\Re(A)^{-1}+\Re(B)^{-1}}{2}\right)\right\|^{2} \quad(\text { by Lemma 2.2) } \\
& \leq \frac{1}{4}\left\|\sec ^{2}(\alpha) M m \Phi\left(\Re\left(\frac{A+B}{2}\right)\right)+\sec ^{2}(\alpha) \Phi\left(\frac{\Re(A)^{-1}+\Re(B)^{-1}}{2}\right)\right\|^{2} \quad \text { (by (6)) }
\end{aligned}
$$




$$
\begin{aligned}
& =\frac{1}{4}\left\|\sec ^{2}(\alpha) \Phi\left(M m \Re\left(\frac{A+B}{2}\right)+\frac{\Re(A)^{-1}+\Re(B)^{-1}}{2}\right)\right\|^{2} \\
& \leq \frac{1}{4} \sec ^{4}(\alpha)(M+m)^{2} \quad(\text { by }(31)) .
\end{aligned}
$$

That is,

$$
\left\|\Phi(\Re(A \sharp B)) \Phi^{-1}\left(\Re\left(\left(\frac{A^{-1}+B^{-1}}{2}\right)^{-1}\right)\right)\right\| \leq \sec ^{2}(\alpha) K(h) .
$$

This completes the proof.

\section{Funding}

This research is supported by the National Natural Science Foundation of P.R. China (No. 11571247).

\section{Competing interests}

The authors declare that they have no competing interest.

\section{Authors' contributions}

All authors contributed almost the same amount of work to manuscript. All authors read and approved the final manuscript.

\section{Publisher's Note}

Springer Nature remains neutral with regard to jurisdictional claims in published maps and institutional affiliations.

Received: 7 January 2019 Accepted: 15 April 2019 Published online: 27 April 2019

\section{References}

1. Bhatia, R.: Positive Definite Matrices. Princeton University Press, Princeton (2007)

2. Bhatia, R., Kittaneh, F.: Notes on matrix arithmetic-geometric mean inequalities. Linear Algebra Appl. 308, 203-211 (2000)

3. Drury, S.: Principal powers of matrices with positive definite real part. Linear Multilinear Algebra 63, 296-301 (2015)

4. Drury, S., Lin, M.: Singular value inequalities for matrices with numerical ranges in a sector. Oper. Matrices 8 , $1143-1148(2014)$

5. Fu, X., Liu, Y., Liu, S.: Extension of determinantal inequalities of positive definite matrices. J. Math. Inequal. 11, 355-358 (2017)

6. Lin, M.: Squaring a reverse AM-GM inequality. Stud. Math. 215, 187-194 (2013)

7. Lin, M.: On an operator Kantorovich inequality for positive linear maps. J. Math. Anal. Appl. 402, 127-132 (2013)

8. Lin, M.: Extension of a result of Haynsworth and Hartfiel. Arch. Math. 104, 93-100 (2015)

9. Lin, M.: Some inequalities for sector matrices. Oper. Matrices 10, 915-921 (2016)

10. Lin, M., Sun, F.: A property of the geometric mean of accretive operators. Linear Multilinear Algebra $65,433-437$ (2017)

11. Liu, J., Wang, Q.: More inequalities for sector matrices. Bull. Iran. Math. Soc. 44, 1059-1066 (2018)

12. Marshall, A.W., Olkin, I.: Matrix versions of Cauchy and Kantorovich inequalities. Aequ. Math. 40, 89-93 (1990)

13. Tominaga, M.: Specht's ratio in the Young inequality. Sci. Math. Jpn. 55, 583-588 (2002)

14. Yang, C., Lu, F.: Some generalizations of inequalities for sector matrices. J. Inequal. Appl. 2018, 183 (2018)

15. Zhang, P.: More operator inequalities for positive linear maps. Banach J. Math. Anal. 9, 166-172 (2015)

\section{Submit your manuscript to a SpringerOpen ${ }^{\circ}$ journal and benefit from:}

- Convenient online submission

- Rigorous peer review

- Open access: articles freely available online

- High visibility within the field

- Retaining the copyright to your article

Submit your next manuscript at $>$ springeropen.com 